\title{
RESEARCH
}

Open Access

\section{The chicken gut metagenome and the modulatory effects of plant-derived benzylisoquinoline alkaloids}

Peng Huang ${ }^{1,2+}$, Yan Zhang ${ }^{3 \dagger}$, Kangpeng Xiao ${ }^{1,2+}$, Fan Jiang ${ }^{3+}$, Hengchao Wang ${ }^{3}$, Dazhi Tang ${ }^{4}$, Dan Liu ${ }^{4}$, Bo Liu ${ }^{3}$,

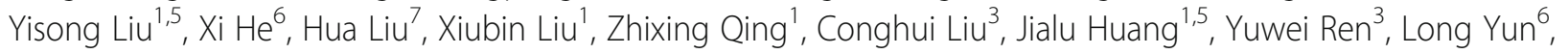
Lijuan Yin ${ }^{3}$, Qian Lin ${ }^{6}$, Cheng Zeng ${ }^{1,5}$, Xiaogang Su ${ }^{5}$, Jingyang Yuan ${ }^{5}$, Li Lin ${ }^{1,5}$, Nanxi Hu ${ }^{1,5}$, Hualiang Cao ${ }^{1,2}$, Sanwen Huang ${ }^{3}$, Yuming Guo ${ }^{4^{*}}$, Wei Fan ${ }^{3 *}$ and Jianguo Zeng ${ }^{1,7^{*}}$

\begin{abstract}
Background: Sub-therapeutic antibiotics are widely used as growth promoters in the poultry industry; however, the resulting antibiotic resistance threatens public health. A plant-derived growth promoter, Macleaya cordata extract (MCE), with effective ingredients of benzylisoquinoline alkaloids, is a potential alternative to antibiotic growth promoters. Altered intestinal microbiota play important roles in growth promotion, but the underlying mechanism remains unknown.

Results: We generated 1.64 terabases of metagenomic data from 495 chicken intestinal digesta samples and constructed a comprehensive chicken gut microbial gene catalog (9.04 million genes), which is also the first gene catalog of an animal's gut microbiome that covers all intestinal compartments. Then, we identified the distinctive characteristics and temporal changes in the foregut and hindgut microbiota. Next, we assessed the impact of MCE on chickens and gut microbiota. Chickens fed with MCE had improved growth performance, and major microbial changes were confined to the foregut, with the predominant role of Lactobacillus being enhanced, and the amino acids, vitamins, and secondary bile acids biosynthesis pathways being upregulated, but lacked the accumulation of antibiotic-resistance genes. In comparison, treatment with chlortetracycline similarly enriched some biosynthesis pathways of nutrients in the foregut microbiota, but elicited an increase in antibiotic-producing bacteria and antibiotic-resistance genes.
\end{abstract}

Conclusion: The reference gene catalog of the chicken gut microbiome is an important supplement to animal gut metagenomes. Metagenomic analysis provides insights into the growth-promoting mechanism of MCE, and underscored the importance of utilizing safe and effective growth promoters.

Keywords: Chicken, Gut metagenome, Microbiome, Growth promoter, Benzylisoquinoline alkaloid, Antibiotic, Chlortetracycline

\footnotetext{
* Correspondence:

guoyum@cau.edu.cn; fanwei@caas.cn; zengjianguo@hunau.edu.cn

${ }^{+}$Peng Huang, Yan Zhang, Kangpeng Xiao and Fan Jiang contributed equally to this work.

${ }^{4}$ State Key Laboratory of Animal Nutrition, College of Animal Science and

Technology, China Agricultural University, Beijing 100193, China

${ }^{3}$ Agricultural Genomic Institute, Chinese Academy of Agricultural Sciences,

Shenzhen 518120, Guangdong, China

${ }^{1}$ Hunan Key Laboratory of Traditional Chinese Veterinary Medicine, Hunan

Agricultural University, Changsha 410128, Hunan, China

Full list of author information is available at the end of the article
}

(c) The Author(s). 2018 Open Access This article is distributed under the terms of the Creative Commons Attribution 4.0 International License (http://creativecommons.org/licenses/by/4.0/), which permits unrestricted use, distribution, and reproduction in any medium, provided you give appropriate credit to the original author(s) and the source, provide a link to the Creative Commons license, and indicate if changes were made. The Creative Commons Public Domain Dedication waiver (http://creativecommons.org/publicdomain/zero/1.0/) applies to the data made available in this article, unless otherwise stated. 


\section{Background}

Global chicken production makes a substantial contribution to food security. Although sub-therapeutic antibiotics have been widely used as growth promoters (AGPs) in livestock to maintain health and enhance productivity, the resulting antibiotic resistance has become a major threat to public health [1]. The European Union has banned the use of AGPs since 2006 [2]; thus, the development of safe alternatives to AGPs has become a global focus.

The growth-promoting mechanisms of AGPs are only partially understood. Germ-free chickens do not gain weight in response to low-dose antibiotic [3]. It is the altered gut microbiota that plays a causal role, not antibiotics, per se [4]. The effects of AGPs are generally thought to be through inhibition of sub-clinical infections, reduction of growth-depressing metabolites from gut microbiota, reduction of nutrients available for pathogens, and so forth [5]. However, it is still unclear how sub-therapeutic antibiotics could efficiently prevent infection and promote growth, and hence, further studies are needed to advance our understanding of AGPs.

Natural growth promoters (NGPs), such as probiotics, prebiotics, and phytobiotics, have been exploited, as alternatives to antibiotics in livestock production. In fact, most NGPs take effect through altering the gut microbiota. Probiotics are living microorganisms that confer benefits to the host, examples being Lactobacillus and Bifidobacterium [6]. Prebiotics are substrates selectively utilized by gut microbiota and include non-digestible oligosaccharides and polyunsaturated fatty acids [7]. Phytobiotics represent a wide range of plant-derived bioactive compounds, which confer multiple effects to the host, and can also stimulate beneficial bacteria in the gut [8].

The phytobiotic Macleaya cordata extract (MCE) has been widely used, for decades, in feed livestock in many countries [9]. The effective chemical composition of MCE includes sanguinarine and chelerythrine, both belonging to a group of benzylisoquinoline alkaloids, which have antimicrobial and anti-inflammatory properties [10-12]. Additionally, sanguinarine has a molecular structure highly similar to another benzylisoquinoline alkaloid, berberine, which is clinically effective in treating some diseases by modulation of the gut microbiota [13, 14]. Nevertheless, details concerning the mechanism(s) associated with growth promotion remain unclear.

Currently, the gut microbial gene catalogs of humans, mice, and pigs have been established [15-18], which greatly facilitated gut microbial studies in the health and diseases in these hosts. In this study, we constructed the first comprehensive chicken gut microbial gene catalog, to better understand the related microbiota. We then systematically studied the impact of MCE and the commonly used AGP, chlortetracycline
(CTC). The gut metagenome analysis provided a deeper insight into the growth promoters.

\section{Results and discussion}

A comprehensive chicken gut microbial gene catalog and its comparison with the human and pig catalogs

We collected 495 digesta samples from the five intestinal compartments (duodenum, jejunum, ileum, cecum, and colorectum) of chickens raised in 7 different farms in China. These chickens varied in breeds (7 breeds: HyLine Variety Brown, Cobb 500, Ross 308, Arbor Acres broiler, Local yellow-feather chickens, Yellow dwarf chicken, and Guangxi local chicken), farming systems (battery-cage and free-range), and farm location (6 Chinese provinces) (Fig. 1a and Additional file 1: Table S1). High-throughput sequencing generated a total of 1.64 terabases $(\mathrm{Tb})$ of clean metagenomic data, without low-quality reads, adapter or host contaminants, with an average of 3.31 gigabases (Gb) per sample. Based on the assembled contigs with an N50 contig length of $1.95 \mathrm{~kb}$, we identified 9.04 million non-redundant genes, with an average open reading frame (ORF) length of $697 \mathrm{bp}$.

Rarefaction analysis of all samples revealed a curve approaching saturation (Fig. 1b, Additional file 2: Figure S1), suggesting that the vast majority of chicken gut microbial genes are present in our gene catalog. In fact, the size and quality of this chicken gene catalog are comparable to those of human (9.9 million genes) and pig (7.7 million genes) catalogs (Additional file 3: Table S2), which provided useful reference genes for subsequent studies [16, 17]. By comparing the pairwise overlap, at the gene sequence level, we determined that over $80 \%$ of genes are unique to each species, and only a very small percentage $(\sim 0.5 \%)$ of genes are shared by chickens, humans, and pigs. Interestingly, chickens and pigs share fewer microbial genes $(\sim 0.8 \%)$ than do chickens and humans $(\sim 10 \%)$ or pigs and humans $(\sim 10 \%)$ (Fig. 1c), the latter of which is consistent with a previous report [17].

Using CAMRA3 for taxonomic assignment, 80.99\% of the genes in the chicken gut catalog were taxonomically classified at the superkingdom level, among which bacteria account for $98.95 \%$ of the classified genes, with the remaining $1 \%$ being from archaea and eukaryotes. More than $88 \%$ of the bacterial genes are from the top four phyla, including Firmicutes, Actinobacteria, Proteobacteria, and Bacteroidetes (Fig. 1d). In the human and pig gut catalogs, Firmicutes and Bacteroidetes are predominant, and Proteobacteria and Actinobacteria make up a smaller percentage $[16,17]$. At lower taxonomic levels, $25.97 \%$ and $2.29 \%$ of the genes in this catalog were annotated to genus and species, respectively. Note that the short-chain fatty acid (SCFA)-producing genera, such as Bacteroides, Blautia, Ruminococcus, and Faecalibacterium, are among the major genera in both human 


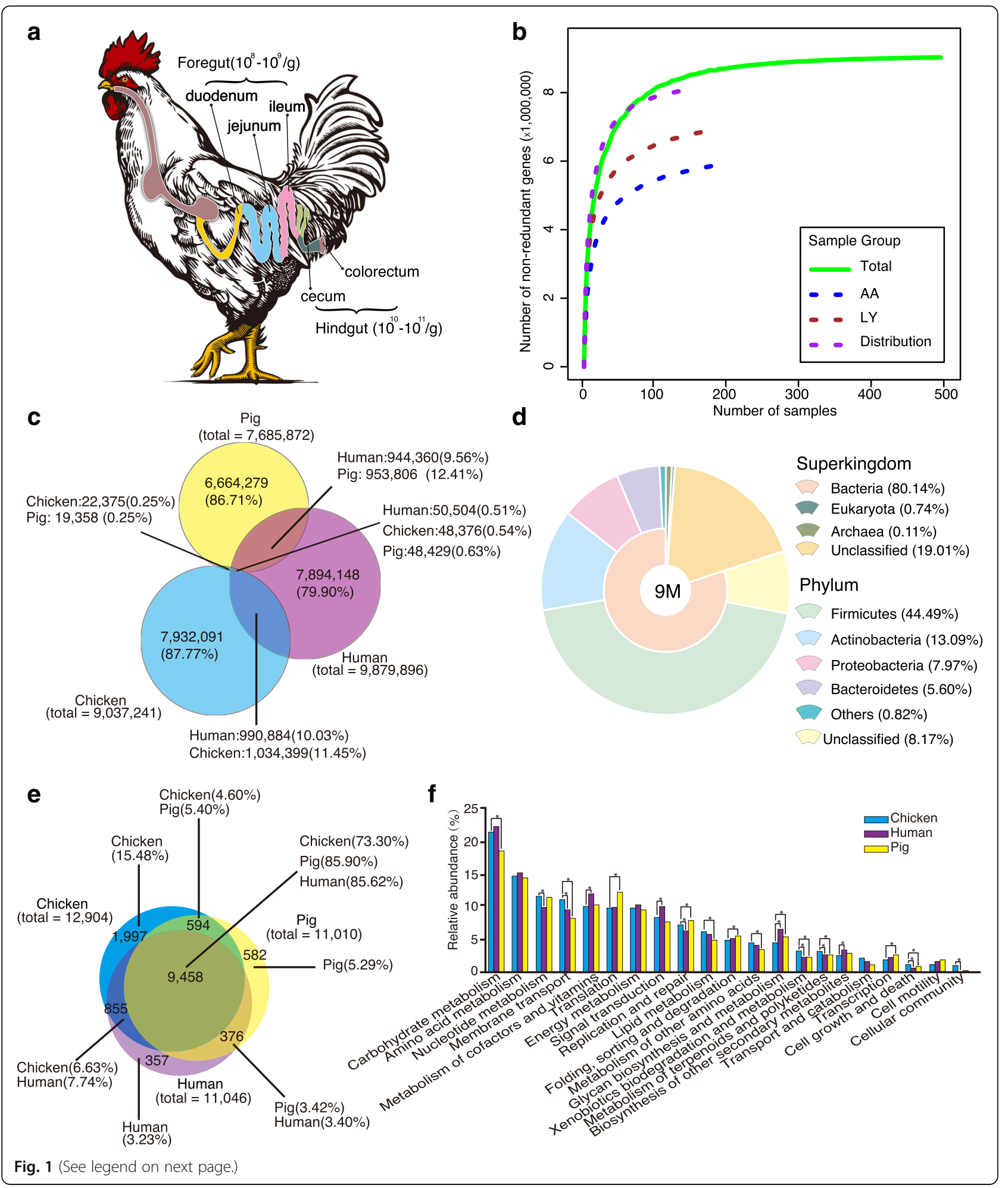


(See figure on previous page.)

Fig. 1 Chicken gut microbial gene catalog. a Diagram of chicken intestinal tract. The microbial densities in the foregut and hindgut were labeled. b Rarefaction curves of detected genes from the whole set of 495 samples (Total) and from subgroups of LY, AA, and Distribution. A total of 9.04 million non-redundant genes were detected, and the rarefaction curve including all samples approaches saturation at the end of sampling. The gene number of a specific number of samples was calculated after random samplings repeated 100 times with replacement, and the median was plotted. c Venn diagram of gut microbial genes shared between the chicken, human, and pig catalogs. The criteria for shared genes were sequence identity $>95 \%$ and overlap $>90 \%$ of the shorter gene. $\mathbf{d}$ Taxonomic annotation of the chicken gut gene catalog at the superkingdom and phylum levels. e Venn diagram of KEGG orthologous groups (KOs) present in and shared by chicken, human, and pig catalogs. $\mathbf{f}$ Comparison of KEGG functional profiles (relative gene abundance summarized into KEGG functional categories and genes without functional annotations were excluded) of gut microbiome among chickens, humans, and pigs. Asterisks denote Wilcoxon rank-sum test result $(P<0.005)$

and pig guts. Similarly, in the chicken gut, these genera are also among the major genera of relatively high abundance (Fig. 2d, Additional file 4: Table S3), indicating the importance of these gut microbes in both birds and mammals.

Using KEGG and eggNOG for function gene classification, 5,454,369 (60.4\%) and 6,881,483 (76.1\%) genes in the catalog were annotated with KEGG orthologous groups (KOs) and eggNOG orthologous groups (OGs), respectively; values are comparable to those of human and pig catalogs (Additional file 5: Figure S2). As shown by Venn diagrams (Fig. 1e and Additional file 6: Figure S3), a large majority of the KOs (73-86\%) and OGs (4677\%) were shared among chickens, humans, and pigs, representing shared gut microbial functions, despite vast differences at the gene sequence level.

The KEGG functional profiles, based on the functional assignments and relative gene abundances, also showed similarities in gut microbial functions in the different hosts (Fig. 1f). However, there were still significant differences $(P<0.005)$ in some KEGG functional categories, and the non-metric multidimensional scaling (NMDS) analysis of KOs also showed clear differences among chicken, human, and pig gut samples (Additional file 7: Figure S4). Notably, the genes for glycan biosynthesis and metabolism were more abundant in human and pig guts, whereas the genes for membrane transport, an essential mechanism for the uptake of substrates, such as sugars, lipids, peptides, and ions, were more abundant in the chicken gut microbiota (Fig. 1f). The enriched genes for membrane transport likely reflect the availability of more nutrient substrates, in the chicken intestine, for direct microbial utilization. The more abundant genes for metabolism of xenobiotics, terpenoids, and polyketides, in the chicken gut, are relevant to the abundant bacterial phylum Actinobacteria, which decomposes organic matter and produces various natural drugs, enzymes, and bioactive metabolites [19].

With the first comprehensive chicken gut catalog and the diverse samples from different farms (DHC, DGY, DHK, DSL, and DST) (Additional file 1: Table S1), we determined that the taxonomic variability among samples was high (Additional file 8: Figure S5), and the NMDS plot showed separations among chicken groups from different farms (Additional file 9: Figure S6). Notably, the intestinal microbial diversities (Shannon index) of chickens in free-range farming (DHC and DGY) were higher than those in battery-cage (DHK, DSL and DST) systems (Additional file 10: Figure S7). In addition, Actinobacteria, which is a dominant soil phylum, was more abundant in free-range farming than in battery-cage chickens (Additional file 11: Figure S8). The observed differences were associated with lifestyles, as free-range chickens were exposed to the outdoor environment and came into contact with more diverse microbes that shape their different gut microbiota.

\section{Distinctive characteristics of chicken foregut and hindgut metagenomes}

Distinguished by the difference in morphology and function, the chicken intestinal tract can be divided into the foregut and hindgut. The foregut contains the duodenum, jejunum, and ileum compartments. The duodenum has the major function in feed digestion by using digestive enzymes and bile from the pancreas and liver, and the released nutrients, such as amino acids, fatty acids, sugars, and peptides, are mainly absorbed in the jejunum and ileum. The hindgut contains the cecum and colorectum. Substantial microbial fermentation occurs in the cecum, which provides nutrients, detoxifies some harmful substances, and also helps to prevent pathogen colonization $[20,21]$. The colorectum is the distal part of the intestinal tract, where residual water and salt absorption occurs [22]. Previous chicken gut studies focused more on cecal or fecal microbiota [23-25], and some microbial functions such as polysaccharide utilization, SCFAs production, and hydrogen consumption in the cecum have been studied [26, 27]. The microbiota in the foregut where nutrient absorption primarily occurs were mostly studied by $16 \mathrm{~S}$ rRNA gene sequencing with limited sample size [28, 29]. In this part of our study, metagenomic data of 285 samples from all five intestinal compartments in chickens older than 40 days were analyzed (Fig. 1a and Additional file 1: Table S1).

Based on the relative abundance of genes, genus, OGs, and KOs, we examined the microbial diversities (Shannon indexes) in each intestinal compartment 

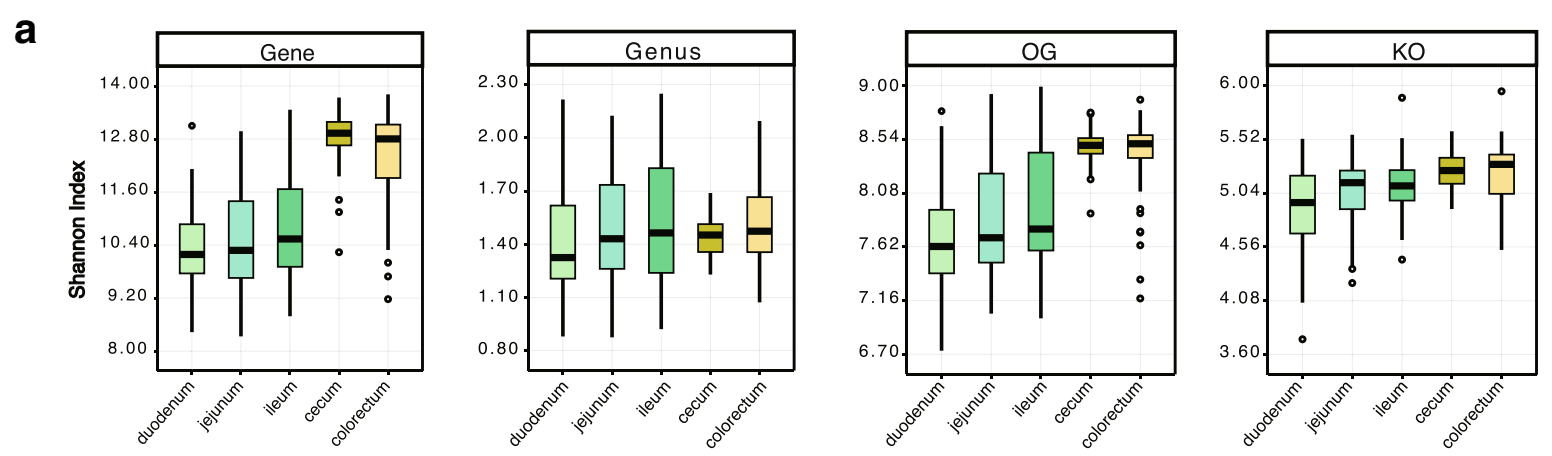

b

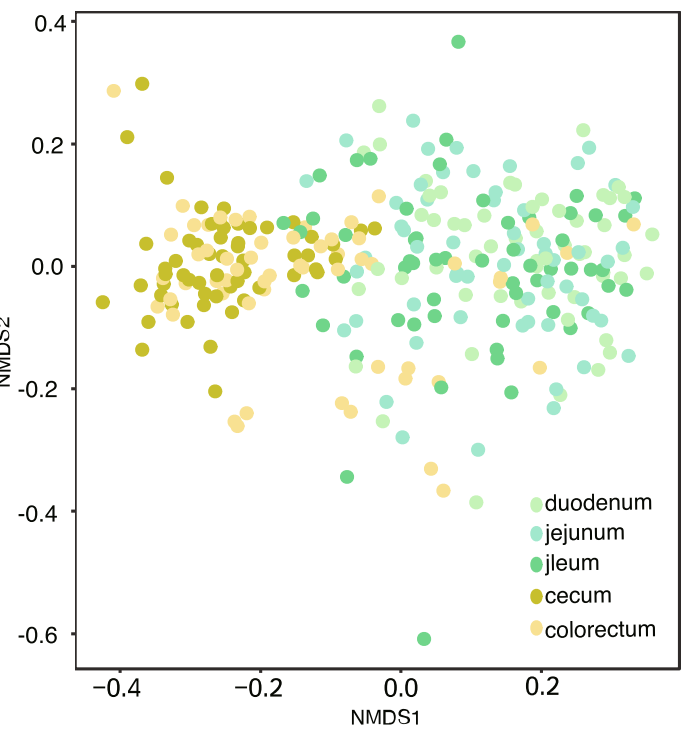

d

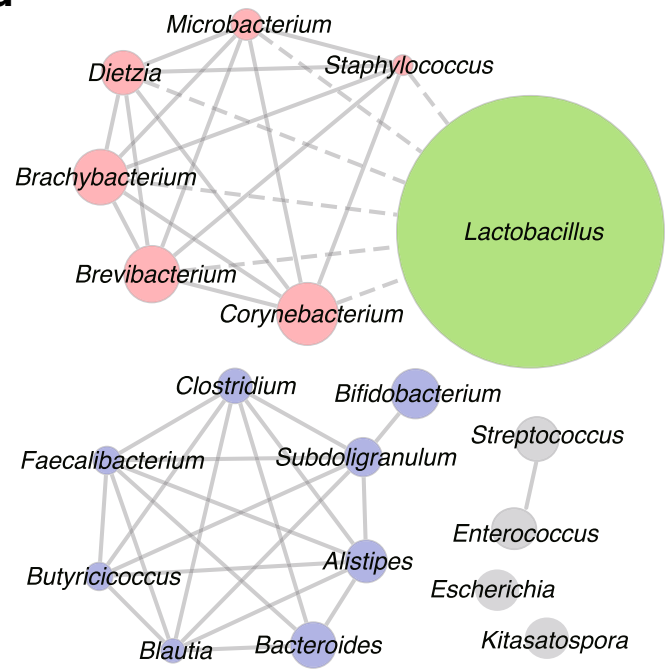

C

$$
\square \text { Foregut } \square \text { Hindgut }
$$

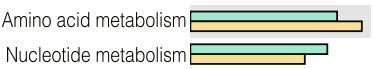

Energy metabolism

$$
\text { Translation }
$$

Metabolism of cofactors and vitamins Signal transduction Lipid metabolism Membrane transport

Biosynthesis of other secondary metabolites Replication and repair

Metabolism of other amino acids Metabolism of terpenoids and polyketides Xenobiotics biodegradation and metabolism

Glycan biosynthesis and metabolism Transcription $\square$

Folding, sorting and degradation Signaling molecules and interaction
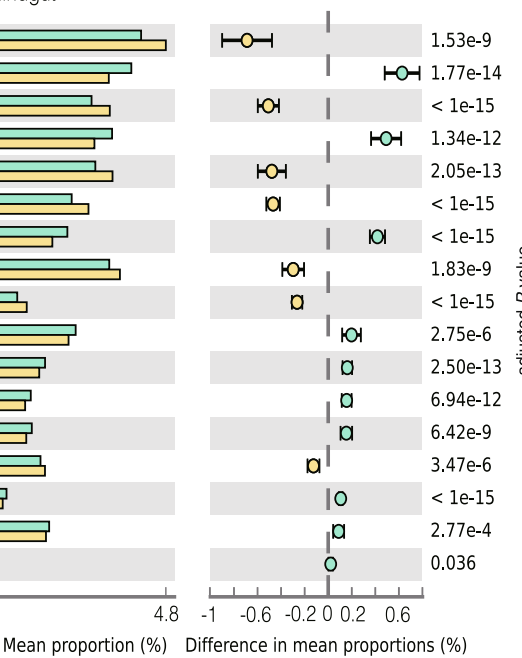

e

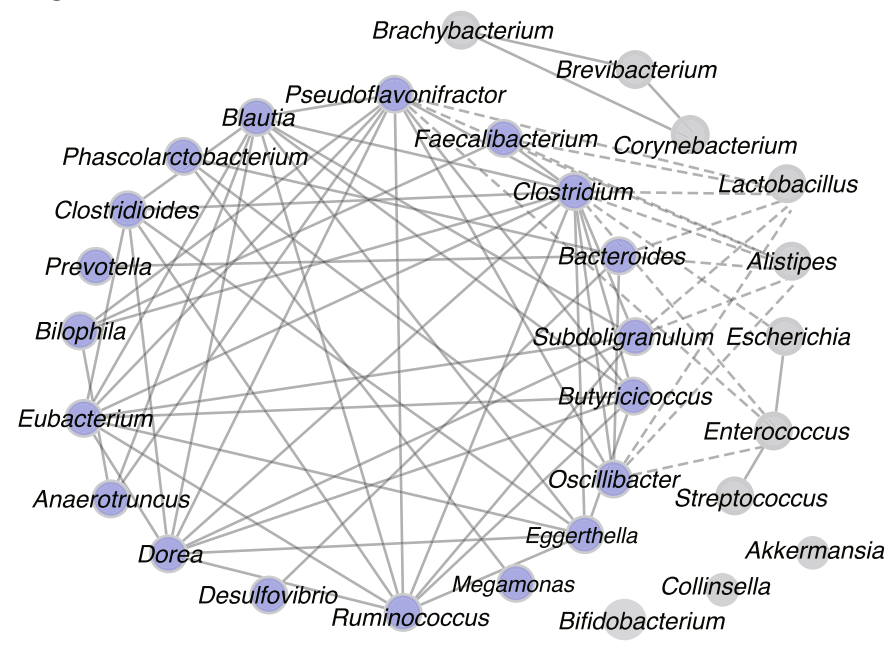

Fig. 2 (See legend on next page.) 
(See figure on previous page.)

Fig. 2 Comparison of gut microbiome in different intestinal compartments of chickens. a Microbial diversity (Shannon index) at gene, genus, OG, and $\mathrm{KO}$ levels. Box plots show median \pm interquartile range (IQR) and 1.5 IQR ranges (whiskers), with outliers denoted by dots. $\mathbf{b}$ The non-metric multidimensional scaling (NMDS) plot based on Bray-Curtis dissimilarities at species level. An obvious difference was observed between the foregut (duodenum, jejunum, and ileum) and hindgut (cecum and colorectum). c Differences in microbial functions between the foregut and hindgut based on KEGG functional categories (Wilcoxon rank-sum test, Storey's methods for multiple tests adjustment). Chicken gut microbial co-occurrence network analysis based on core genus (average relative abundance $>0.1 \%$ ) $\mathbf{d}$ in the foregut and $\mathbf{e}$ hindgut. Solid line:

Spearman's rank correlation coefficient $>0.30$; dash line: Spearman's rank correlation coefficient $<-0.30$. The size of nodes was proportional to the relative abundance of genera

(Fig. 2a). Our results indicated that microbial diversities in the foregut compartments were approximately the same, with only a slight increase from duodenum, jejunum, to ileum. A similar situation was observed between the hindgut cecum and colorectum; however, the diversities were clearly higher in the hindgut than in the foregut compartment (Fig. 2a).

We next calculated the number of common genes presented in $50 \%$ of the samples from each intestinal compartment, and the results showed that the common genes accounted for only about $2 \%$ of all genes in each foregut compartment (duodenum, jejunum, and ileum), but the number increased to $18 \%$ and $13 \%$ in the cecum and colorectum, respectively (Additional file 12: Table S4). The NMDS analysis revealed that there was a clear separation between the foregut and hindgut compartments (Fig. 2b). Taken together, these results indicated an overall similarity within foregut and hindgut compartments, with a larger difference between them, which is consistent with previous studies [29, 30].

The relative abundance profiles of phylum and genus showed distinct microbial features between the foregut and hindgut samples (Additional file 8: Figure S5). At the phylum level, Actinomycetes and Bacteroides showed significant differences $(P<0.001)$ and were twofold enriched in the foregut and sixfold enriched in the hindgut, respectively (Additional file 4: Table S3). As previously reported [29, 31], at the genus level, Lactobacillus is the predominant genus in the foregut, but not in the hindgut (Additional file 4: Table S3); Lactobacillus provides nutrients to the host and defends against opportunistic pathogens [32, 33]. Moreover, the relative abundance of genera, such as Corynebacterium, Brevibacterium, and Brachybacterium, in the foregut was higher than that in the hindgut (Additional file 4: Table S3). By contrast, a variety of anaerobic genera, such as Subdoligranulum, Bacteroides, Faecalibacterium, Clostridium, and Butyricicoccus, were more abundant in the hindgut (Additional file 4: Table S3).

By using the 285 samples from chickens older than 40 days, we constructed the co-occurrence network of the core genera in both foregut and hindgut. In the foregut, Lactobacillus competitively inhibits a cluster of bacteria, with negative correlations with all these genera
(Fig. 2d). Additionally, some SCFA producers, such as Clostridium, Butyricicoccus, and Faecalibacterium, showed positive correlations with one another and form a relatively independent and stable cluster (Fig. 2d). In the hindgut, 19 genera are positively correlated with each other and form a large co-occurrence network (Fig. 2e). Some of these bacteria are beneficial intestinal microbes that produce metabolites, such as SCFAs, by fermentation [26, 34], while opportunistic pathogenic bacteria, Escherichia and Enterococcus, are inhibited by the central microbial cluster (Fig. 2e). These results revealed more diverse and complex microbial communities in the hindgut than in the foregut.

According to our KEGG functional analysis (Fig. 2c and Additional file 13: Figure S9), the microbiota in the foregut was enriched in genetic information processing for transcription, translation, and replication, as well as the metabolic functions of nucleotides and lipids, whereas microbes in the hindgut were enriched for the metabolic functions of amino acids, energy metabolism, and secondary metabolite biosynthesis. Similar results were obtained based on eggNOG analysis (Additional files 14 and 15: Figure S10 and S11). These findings were consistent with the substantial microbial fermentation in the hindgut, and the production of various metabolites, such as amino acids and SCFAs that are important for host health [3537]. By taking the taxonomic features into consideration, we noticed that Lactobacillus has a relatively small genome (2 Mb) compared to other bacteria, and encodes higher proportion of genes for basic functions such as transcription, translation, and replication, but lower proportion of genes for many diverse metabolic functions. Therefore, the predominance of Lactobacillus in the foregut has largely contributed to the functional differences between the foregut and hindgut microbiome. In conclusion, the taxonomic and functional features of the foregut and hindgut microbiome are consistent with the morphological and physiological structure of the chicken intestine.

\section{Temporal development and maturation of the chicken gut microbiome}

To investigate the development of gut microbial communities, samples of five different chicken ages $(1,7,14$, 


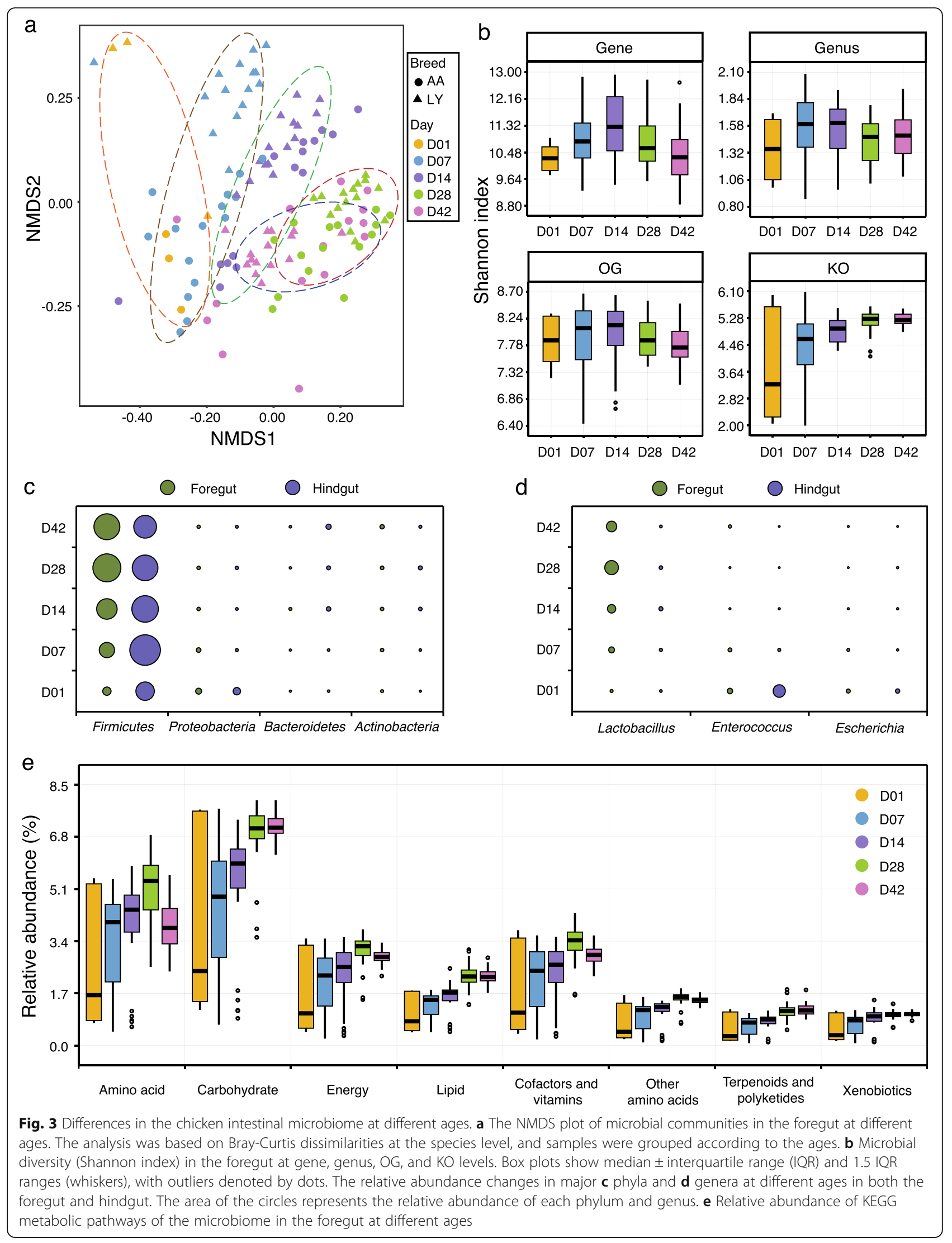


28, and 42 days) were analyzed for two breeds, Arbor Acres (AA) and Local yellow-feather (LY). Previously, researchers have found that the microbiota were inherited partly from maternal hens, and then influenced by environmental factors [38]. Our data showed that the gut microbiome of newly hatched chicks (day 1 ) were highly variable and obviously different from other samples (day 7, 14, 28, and 42) (Fig. 3), reflecting the shortterm exposure to the environment and the initiation of gut microbial communities.

The microbial development is influenced by many factors, such as diet, feed additive, and host breed, and the successional changes have been reported for a few chicken breeds [39-41]. In our study, for both AA and LY chickens, the NMDS plots showed that samples were clustered into groups by ages, with day 28 and 42 groups exhibiting much higher similarity (Fig. 3a and Additional file 16: Figure S12), which also revealed a successional development. Microbial diversities increased during chicken development, peaking at approx. day 14 and 28 for the foregut and hindgut, respectively, and then remaining stable or decreasing slightly thereafter (Fig. 3b and Additional file 17: Figure S13). Both the NMDS and microbial diversity analyses indicated that the intestinal microbiota develops into a relatively mature community, as the host chicken matures, and then, a stable state is maintained.

Firmicutes, Proteobacteria, Bacteroidetes, and Actinobacteria were the dominant phyla in both foregut and hindgut throughout the growth test, and they exhibited obvious temporal changes (Fig. 3c). The most abundant phylum, Firmicutes, increased in the foregut from day 1 to day 28 , and then remained relatively stable until day 42 , whereas in the hindgut, it slowly decreased from day 7 to day 42 (Fig. 3c and Additional file 18: Table S5). The predominant genus in the foregut, Lactobacillus (phylum: Firmicutes), changed in a similar way to Firmicutes (Fig. 3d and Additional file 19: Table S6). In the hindgut, major genera, such as Lactobacillus, Subdoligranulum, and Bifidobacterium, were more abundant in the middle growth period, while other genera, such as Alistipes, were more abundant at the end of the growth test (Additional file 20: Figure S14). In addition, the frequently reported zoonotic pathogens such as Salmonella and Campylobacter could be detected in both foregut and hindgut throughout the growth test, but for the reason that only healthy chickens were studied, both of the pathogens were detected at very low levels (average relative abundance, $0.01-0.03 \%$ ) and their impacts might be much weaker than those in the infected chickens [42, 43]. In both foregut and hindgut, the metabolic capacity reached a maximum at day 28 , thereafter remaining stable. However, differences among the ages were larger in the foregut than those in the hindgut (Fig. 3e and Additional file 21: Figure S15). The findings showed that the early days are critical both for chicken development and establishment of the gut microbiota.

\section{CTC and MCE promote chicken growth}

To examine the effects of MCEs, compared with antibiotics, we next performed parallel experiments with two chicken breeds, each with five test groups that received CTC supplementation and three MCE gradient dosages (MCE-L, MCE-M, MCE-H), as feed additives, as well as a blank control (BLANK). The LY and AA chicken breeds were independently raised on two farms and measurements taken at 56 and 42 days, respectively.

In LY chickens (day 56), dietary supplements numerically (not significantly) improved the average body weight gain by $3.1 \%$ (CTC, $P=0.258$ ), 2.2\% (MCE-L, $P$ $=0.204), 4.8 \%$ (MCE-M, $P=0.071)$, and 3.1\% (MCE-H, $P=0.069)$, and the feed intakes were also significantly $(P<0.05)$ increased by $3.0-6.9 \%$ (Table 1$)$. Therefore, the feed conversion ratio (FCR) was not significantly changed $(P>0.05)$. In AA chickens (day 42$)$, the average body weight gain was also numerically (not significantly) improved by $1.5 \%$ (CTC, $P=0.363), 2.2 \%$ (MCE-M, $P=0.258)$, and $1.7 \%$ (MCE-H, $P=0.302)$ (Table 1). However, the increase of feed intake in AA chickens was not significant $(P>0.05)$. As a result, the FCR was significantly decreased $(P<0.05$, from 1.77 to 1.71 in MCE-M), indicating the improvement of the nutrient absorption, which benefits the chicken farming. These

Table 1 Chicken growth performance in response to CTC and MCE treatments

\begin{tabular}{|c|c|c|c|c|c|c|}
\hline \multirow[t]{2}{*}{ Group } & \multicolumn{3}{|c|}{ Local yellow-feather chickens (day 56) } & \multicolumn{3}{|c|}{ Arbor Acre chickens (day 42) } \\
\hline & Feed intake (g) & Body gain (g) & Feed conversion ratio & Feed intake $(\mathrm{g})$ & Body gain (g) & Feed conversion ratic \\
\hline BLANK & $3443.13 \pm 37.87^{b}$ & $1505.37 \pm 49.46$ & $2.33 \pm 0.05$ & $4462.13 \pm 73.48$ & $2519.13 \pm 28.14$ & $1.78 \pm 0.02^{\mathrm{a}}$ \\
\hline CTC & $3547.64 \pm 63.51^{\mathrm{a}}$ & $1551.70 \pm 39.45$ & $2.31 \pm 0.05$ & $4522.67 \pm 80.36$ & $2557.41 \pm 40.17$ & $1.77 \pm 0.01^{\mathrm{a}}$ \\
\hline MCE-L & $3597.37 \pm 45.27^{\mathrm{a}}$ & $1538.34 \pm 42.02$ & $2.32 \pm 0.79$ & $4437.84 \pm 89.70$ & $2510.00 \pm 45.12$ & $1.77 \pm 0.01^{\mathrm{a}}$ \\
\hline MCE-M & $3681.41 \pm 126.91^{a}$ & $1577.95 \pm 83.28$ & $2.37 \pm 0.06$ & $4405.79 \pm 88.12$ & $2575.28 \pm 45.66$ & $1.71 \pm 0.01^{b}$ \\
\hline MCE-H & $3627.05 \pm 184.14^{\mathrm{a}}$ & $1551.34 \pm 27.37$ & $2.31 \pm 0.07$ & $4489.43 \pm 73.73$ & $2562.08 \pm 38.05$ & $1.75 \pm 0.01^{\mathrm{a}}$ \\
\hline$P$ value & 0.018 & 0.163 & 0.361 & 0.869 & 0.728 & 0.004 \\
\hline
\end{tabular}

Data are presented as mean $\pm S D$; data in columns with no common superscript differ significantly $(P<0.05)$. Feed conversion ratio (feed intake/weight gain). Data for growth performance were analyzed with one-way ANOVA and Duncan's multiple comparison in SPSS Version 18.0 (SPSS Inc., Chicago, Illinois, USA) 
results are consistent with previous findings that CTC and MCE could promote the body weight gain $(2-5 \%)$, by improving either food intake or feed conversion efficiency [44-47]. However, the effects of MCE and CTC appeared more pronounced for large-scale chicken farms, probably due to the differences in farming conditions. In addition, chicken breeds, diets, and other factors also influence the effects of CTC and MCE [44-46], suggesting the complex mechanisms on growth promotion.

\section{CTC and MCE reshape the chicken foregut microbiota to promote growth}

Major microbial responses to growth promoters were observed in the foregut, but not in the hindgut (Fig. 4, Additional files 22, 23, 24, 25, 26, and 27: Figures S16-S21 and Additional file 28: Table S7). This is consistent with a previous finding that treatment with an AGP, avilamycin, more strongly impact microbiota composition in the ileum than in the cecum [48]. Considering that the hindgut has a much higher microbial cell density and microbial diversity (Figs. 1a and 2a) [49], as well as a more complex microbial network compared to the foregut (Fig. 2d, e), the microbiota in the hindgut is more stable and less affected by feed additives. In addition, the chicken ceca are a pair of blind-ended pouches, which provide a relatively closed microbial environment, and thus, are more likely to be resilient to interference. Our findings support the notion that microbial regulation, by growth promoters, in the foregut plays a more important role.

In the foregut, the predominant genus, Lactobacillus, was influenced by MCE. The average relative abundance was increased by $12-54 \%$ in the three groups (Fig. $4 \mathrm{~b}$ and Additional file 24: Figure S18a), and thus, the predominant role of Lactobacillus in the foregut was further strengthened, particularly by MCE-H. Lactobacillus is recognized as a beneficial probiotic that produces vitamins and organic acids, and also competitively inhibits pathogens [33, 50, 51]. Moreover, through the "cross-feeding" mechanism, the lactate produced by Lactobacillus could be used by anaerobic bacteria to produce butyrate [52], which is an important energy source for intestinal cells and exerts anti-inflammatory activities [53]. In the CTC group, Lactobacillus was increased $(P>0.05)$ by $4 \%$, but this increase was lower than that in the MCE groups, and there were some inconsistencies regarding the impact of CTC on Lactobacillus $[54,55]$. By contrast, CTC significantly $(P<0.05)$ enriched the antibiotic-producing genera of Kitasatospora and Streptomyces (Fig. 4b and Additional file 24: Figure S18a), which are both from the family Streptomycetaceae and produce a variety of antibiotics $[56,57]$. In particular, $80 \%$ of currently used antibiotics are sourced from Streptomyces [57]. Consistent with these findings, we observed the enrichment of several antibiotic biosynthesis pathways in the CTC group (Additional file 24: Figure S18b), including those for tetracycline, macrolides, type II polyketide, and clavulanic acid. The presumably enhanced production of natural antibiotics would amplify the antimicrobial and anti-inflammatory effects of the administered antibiotic, and therefore benefit the host. On the other hand, however, we established that the antibiotic resistance genes (ARGs) were also increased $(P=0.097)$ in the CTC group (Fig. 4c and Additional files 24 and 25: Figures S18c and S19). These findings provide a new perspective to understand the complex impact of sub-therapeutic antibiotic treatment.

The relative abundances of some core genera, such as Corynebacterium, Brachybacterium, and Dietzia, which were in the same microbial co-occurrence network in the foregut (Fig. 2d), were significantly decreased in both MCE and CTC groups (Additional file 26: Figure S20). By comparing the three MCE groups, we found that the high dose had stronger inhibiting effect, and more genera were significantly $(P<0.05)$ decreased in MCE-H than in MCE-M and MCE-L (Additional file 26: Figure S20). This effect might be associated with the competitive inhibition effect of Lactobacillus (Fig. 2d), and the higher relative abundance of Lactobacillus in MCE- $\mathrm{H}$. Most of the inhibited genera by MCE (Additional file 26: Figure S20) are normal, with no clear benefit or harm to the host, whereas genera such as Corynebacterium and Microbacterium also include pathogenic species that may severely threaten animal health $[58,59]$. The decrease of pathogens is likely to alleviate the host inflammation and immune responses, and indeed, our data showed that the host cytokines including IL-4, IFN- $\gamma$, and NF- $\mathrm{kB}$ were downregulated (Additional file 29: Table S8). However, the modulatory effects of MCE were possibly not associated with the frequently reported pathogens such as Salmonella or Campylobacter [42, 43, $60]$, as their relative abundances were low in samples and little change was found after the MCE treatment. In addition, many other bacteria at similar or lower abundance levels may be involved in the modulation as well, which made the detailed mechanism more complicated. Overall, the positive regulation of beneficial Lactobacillus, and the negative regulation of some commensal and pathogenic bacteria, constitutes the overall foregut microbial compositional changes after the MCE treatment.

MCE significantly $(P<0.05)$ enriched the amino acid biosynthesis and metabolism pathways in the foregut (Fig. 4d). Microbial-synthesized amino acids are an important nutrient supplement for the host, and these molecules are primarily absorbed in the foregut rather than in the hindgut [61]. Dietary supplementation of amino acids, such as lysine and arginine, improved the body weight and feed conversion efficiency, as well as 


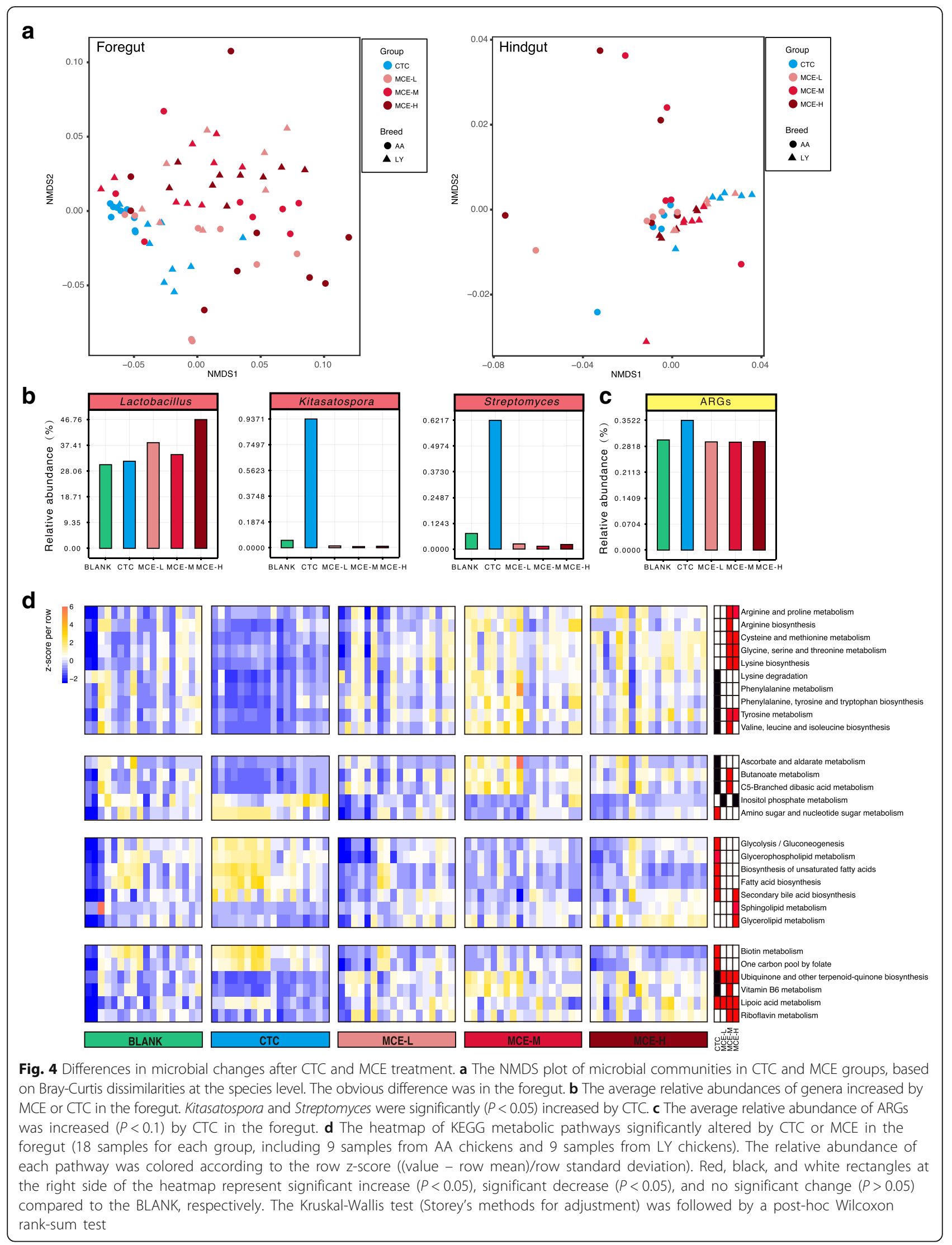


enhanced the immunity $[61,62]$. Accordingly, the metagenomic results suggest that the growth promotion may be achieved by the enhancement of microbial amino acid biosynthesis.

In lipid metabolism, the secondary bile acid biosynthesis pathway was enriched by both MCE and CTC. The host-secreted bile acids have antimicrobial activities that alter the gut microbial composition. Meanwhile, the microbial modification of bile acids can facilitate fat absorption, and is therefore involved in regulating host energy metabolism and immune system [63, 64]. Besides, the biosynthesis pathways of fatty acids and unsaturated fatty acids that are closely related to host lipid metabolism were also enriched in the CTC group. This result indicated that the lipid metabolism regulation is an important mechanism for growth promoters.

Moreover, both MCE and CTC influenced the carbohydrate and vitamin metabolism pathways (Fig. 4d). Two sugar-related metabolism pathways were enriched by CTC, while C5-branched dibasic acid and butanoate (butyrate) metabolism pathways were enriched by MCE (Fig. 4d). The SCFA butyrate is a metabolic energy source for intestinal cells, and it has anti-inflammatory effects and helps the host to maintain mucosal barrier integrity $[65,66]$. Vitamins are essential micronutrients for biochemical reactions, and dietary supplement with vitamins enhances the chicken immune system $[67,68]$. The gut microbiota also acts as an important vitamin supplier for the host [33]. In the present study, the biotin and vitamin-like lipoic acid pathways were enriched in the CTC group (Fig. 4d). Particularly, lipoid acid supplements can improve the growth performance and antioxidant capacity of the host [69]. In the MCE groups, a greater variety of pathways were enriched, including lipoic acid, vitamin $B_{6}$, riboflavin, ubiquinone, and other terpenoid-quinone (including vitamin $K_{1}, K_{2}$, and $E$ ) pathways (Fig. 4d). In summary, vitamins, in addition to other microbial synthesized nutrients, were enhanced by MCE and consequently benefited the host.

\section{Conclusions}

Given the importance of chicken production in agriculture and the remarkable contribution of intestinal microbiota to the host's nutrition and health, the chicken gut microbiota has received growing attention worldwide. In the present study, we constructed the first comprehensive gene catalog of the chicken gut microbiome, by using the digesta samples of all intestinal compartments of chickens from diverse farms in China, and of chickens at different ages throughout the growing period of broilers. Importantly, the foregut microbiome was less studied in either humans or other animals. Our metagenomic results emphasized the similarity of the microbiota within the foregut and hindgut compartments, but exhibited distinctive taxonomic and functional differences between them as well. The intestinal microbiota develops into a relative mature community and reaches the maximum metabolic capacity during day 15-28. These findings make an important supplement to the animal gut metagenomes, especially for chickens.

Since the ban on growth-promoting antibiotics in many countries, large-scale chicken farming has faced challenges of prophylaxis and growth promotion. With increasing global consumption of chickens, it has become imperative to develop effective alternatives. Therefore, the well understanding of the growth-promoting mechanism is in great need. Here, we performed the treatment experiment with the dietary supplementation of MCE, and analyzed the changes in chickens as well as in the intestinal microbiome. In general, MCE improved chicken growth performance and modulated intestinal microbiota. Obvious microbial changes were found in the foregut, where nutrients were primarily absorbed, including the increase of beneficial bacteria such as Lactobacillus, and the enrichment of biosynthesis pathways of amino acids, vitamins, and secondary bile acids. Moreover, the increased Lactobacillus competitively inhibited some pathogens, possibly resulting in the alleviation of host inflammation and immune responses. In addition, our analysis also unveils some of the underlying mechanism of CTC, for comparison with MCE (Fig. 5). Taken together, these findings deepen our understanding of growth promoters in livestock, and provide useful information for the development of safe and effective alternatives to AGPs.

\section{Methods}

\section{Chickens, diets and experimental design}

For the distribution groups (Distribution), five chicken breeds from different commercial providers located in Hunan Changde (DHC), Guangdong Yunfu (DGY), Henan Kaifeng (DHK), Shandong Taian (DST), and Shanxi lvliang (DSL), respectively, in China, were included in this study. Three male and three female individuals were included for four chicken breeds (DHC, DGY, DST, DSL), and only three female individuals were included for one breed that is egg-layer (DHK).

In the treatment experiment groups (growth test), chicken breeds of Arbor Acres broiler (AA) and Local yellow-feather (LY) chickens were studied independently, in two farms in Beijing and Hunan Changsha, respectively. Chickens were randomly divided into 5 groups (12 chickens/repeat, 10 repeats/group) for a 42-day (AA) and 56-day (LY) feeding trail, respectively. The treatments were as follows: (1) BLANK, the basal diet; (2) CTC, the basal diet plus antibiotic $(50 \mathrm{mg} / \mathrm{kg} \mathrm{Citifac}$, 


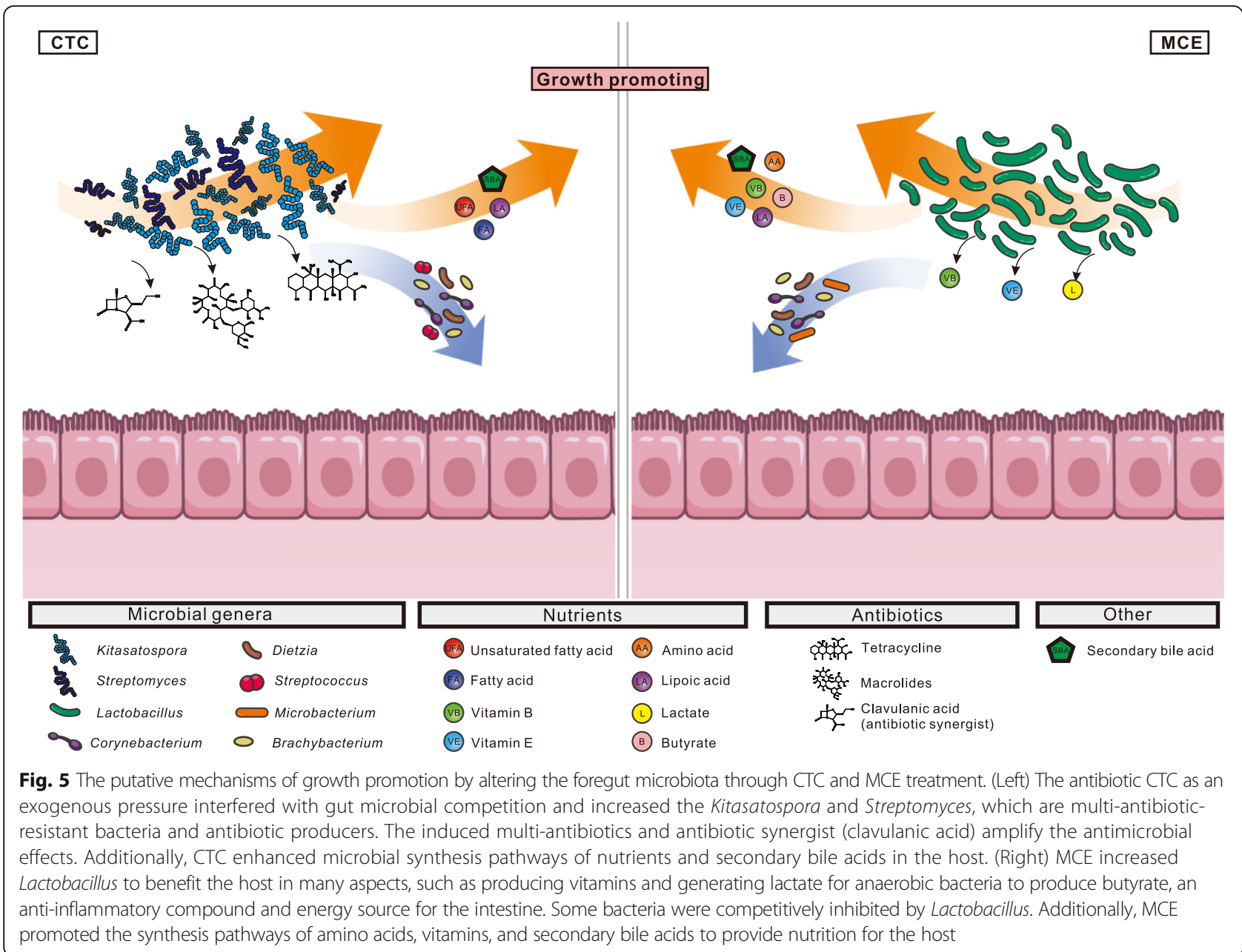

chlortetracycline 20\% $w / w$ premix); (3) MCE-L, the basal diet plus plant extract $\left(15 \mathrm{mg} / \mathrm{kg}\right.$ Sangrovit ${ }^{\circ}$, Macleaya cordata extract $3.75 \% \mathrm{w} / \mathrm{w}$ premix); (4) MCE-M, the basal diet plus plant extract $\left(50 \mathrm{mg} / \mathrm{kg}\right.$ Sangrovit ${ }^{\circ}$, Macleaya cordata extract $3.75 \% \mathrm{w} / \mathrm{w}$ premix); and (5) $\mathrm{MCE}-\mathrm{H}$, the basal diet plus plant extract $(150 \mathrm{mg} / \mathrm{kg}$ Sangrovit, Macleaya cordata extract $3.75 \% \mathrm{w} / \mathrm{w}$ premix). The basal diets were based on the Nutrient Requirements of Poultry: Ninth Revised Edition, 1994 (NRC, 1994) and Feeding Standard of Chicken (NY/T 33-2004). The chickens had free access to feed and water, and were housed in wired three-level battery cages $(100 \mathrm{~cm}$ long $\times 80 \mathrm{~cm}$ wide $\times 40 \mathrm{~cm}$ high/cage) . The lighting schedule was $20 \mathrm{~h}$ light and $4 \mathrm{~h}$ dark throughout the experiment. The room temperature was controlled with heaters and gradually reduced from $35^{\circ}$ $\mathrm{C}$ on day 1 to $24{ }^{\circ} \mathrm{C}$ on day 21 and then kept roughly constant. The chickens were vaccinated using combined Newcastle disease virus (NDV) and infectious bronchitis virus on day 7 through intranasal and intraocular administration, and on day 21 via oral administration. Body weight and feed intake of AA chickens were recorded for each replicate on day 42, while that of LY chickens were recorded for each replicate on day 56.

\section{Collection of intestinal tissue and digesta samples}

To collect intestinal tissue samples of AA chickens (day 21 and day 42), one randomly selected chicken individual from each repeat (ten repeats/group) was slaughtered, and the mid-segment (intestinal tissue) of the ileum was harvested, frozen using liquid nitrogen, and transported to the laboratory in a dry-ice pack, and then kept at $-80^{\circ} \mathrm{C}$ until quantification of gene expression.

To collect intestinal digesta samples, randomly chosen chickens from each group (DST, DSL, DGY, DHC, DHK, $\mathrm{AA}, \mathrm{LY})$ were slaughtered, and then the duodenum, jejunum, ileum, cecum, and colorectum were immediately removed and dissected. Fresh digesta samples were collected, frozen using liquid nitrogen, and transported to the laboratory in a dry-ice pack, then stored at $-80{ }^{\circ} \mathrm{C}$ until DNA extraction. In the treatment experiment groups (growth test), for each intestinal compartment, five digesta samples from five chicken individuals of the same breed (AA and LY chickens), same treatment 
group (BALNK, CTC, MCE-L, MCE-M, and MCE-H) and same age $(7,14,28,42$ days), were pooled as one sample for DNA extraction. The five digesta samples from day 1 chickens before growth promoter treatment were also pooled before DNA extraction. For all the other digesta samples from adult chickens, including 135 samples from distribution groups (DST, DSL, DGY, DHC, DHK) and 150 samples from treatment groups (AA chickens, day 42; LY chickens, day 56), each digesta sample from each individual was processed separately.

\section{RNA isolation and quantitative real-time polymerase chain reaction}

Total RNA from the intestinal tissue was extracted by using Trizol reagent (Invitrogen Life Technologies, Carlsbad, CA) according to the manufacturer's protocol. The concentration and purity of RNA were determined using the NanoDrop 2000 spectrophotometer (Thermo Scientific, Waltham, MA). One microgram of total RNA from each sample was reverse transcribed into cDNA using the PrimeScript RT reagent kit with cDNA eraser (TaKaRa, Dalian, China). The primers used for the reverse transcription were oligo $(\mathrm{dT})$ primer and random hexamers. The one-step real-time PCR was performed with the SYBR ${ }^{\circ}$ Premix Ex TaqTM (TaKaRa, Dalian, China) using a ABI 7500 fluorescence quantitative PCR machine (Applied Biosystems, Foster City, CA) following the manufacturer's guidelines. Primers used in this study were listed in Additional file 30: Table S9. Relative mRNA expression levels of each target gene ( $\beta$-actin, IFN- $\gamma$, IL-4, TNF- $\alpha$, iNOS, NF- $\mathrm{kB}$ ) were calculated using the $2^{-\Delta \Delta C T}$ method.

\section{DNA extraction, library preparation, and sequencing}

The bacteria cells were separated from undigested feed particles and recovered through differential centrifugation before cell lysis [70]. A combination of lysis steps was applied. Cells were subjected to five freeze-thaw cycles (alternating between $65{ }^{\circ} \mathrm{C}$ and liquid nitrogen for $5 \mathrm{~min}$ ), followed by repeated beads-beating in ASL buffer (cat. no. 19082; Qiagen Inc.) plus incubation at $95{ }^{\circ} \mathrm{C}$ for $15 \mathrm{~min}$. DNA was isolated following a previously reported protocol [71]. Metagenomic DNA paired-end libraries were prepared with an insert size of 350 base pairs (bp) following the manufacture's protocol (cat. no. E7645L; New England Biolabs). Sequencing was performed on Illumina HiSeq 2500 and HiSeq X10.

Metagenome assembly and construction of the gene catalog Raw reads were cleaned to exclude adapter sequences, low-quality sequence, as well as contaminated DNA including host and food genomic DNA. The average error rate of the clean reads is lower than 0.001 . The reads that mapped to chicken, human, maize, soybean, wheat, and zebrafish genomes by BWA-MEM were filtered out [72]. Finally, short reads (length $<75$-bp) and unpaired reads were also excluded to form a clean reads data.

For each sample, the clean reads were assembled by Megahit (v1.0.6) under pair-end mode respectively [73], then gene prediction was performed on contigs larger than 500-bp by Prodigal (v2.6.3) with parameter "-p meta" [74], and gene models with cds length less than 102-bp were filtered out. As Megahit is a memory efficient assembly software, in theory, it can assemble reads from all the samples together at once, to improve the assembly result for less abundant species. Here, due to the memory limitation of our computer server, all the 495 samples were firstly divided into 5 study groups: distribution groups (135 samples), treatment groups for LY chickens with 56 days (75 samples), treatment groups for LY chickens with 1-42 days (105 samples), treatment groups for AA chickens with 42 days (75 samples), and treatment groups for AA chickens with 1-42 days (105 samples). Then, assembly and gene prediction were performed on these five study groups independently, using the same methods for each sample.

A non-redundant gene catalog was constructed using the gene models predicted from each sample and each group by cd-hit-est (v4.6.6) [75] with parameter "-c 0.95 -n 10 -G 0 -aS 0.9," which adopts a greedy incremental clustering algorithm and the criteria of identity $>95 \%$ and overlap $>90 \%$ of the shorter genes. By using the gene models predicted from each sample only, we obtained $6 \mathrm{M}$ non-redundant genes; by adding the additional gene models predicted from each group, finally, we obtained a total of $9 \mathrm{M}$ non-redundant genes.

\section{Taxonomic and functional assignment of genes}

Taxonomic assignments of protein sequences were made on the basis of DIAMOND (v0.8.28.90 diamond blastp --evalue 10 --max-target-seqs 250) alignment against the NCBI-NR database by CARMA3 (carma --classify-blast --type p --database p) [76, 77]. A number of 64,332 genes $(0.71 \%)$ classified as eukaryota but not fungi were excluded from the non-redundant gene set, and the final chicken gut gene catalog includes 9,037,241 genes.

The functional assignments of protein sequences were made on the basis of DIAMOND alignment against the KEGG protein database (release 79) and eggNOG (v4.5) $[78,79]$, by taking the best hit with the criteria of $E$ value $<1 \mathrm{e}-5$. The annotation of ARG protein sequences were made on the basis of DIAMOND alignment against the Comprehensive Antibiotic Resistance Database (CARD) [80], with the AMR detection models (protein homolog models) provided by the database.

To calculate of relative gene abundance, the clean reads from each sample were aligned against the gene catalog by BWA-MEM with the criteria of alignment 
length $\geq 50$ bp and identity $>95 \%$. Sequenced-based abundance profiling was performed as previously described [81]. Phylum, genus, species, KO, and OG relative abundances were calculated by summing the abundance of the respective genes belonging to each category per sample, based on the taxonomic assignments, $\mathrm{KO}$ and OG annotations, respectively. The relative gene abundance profile was also summarized into KEGG and eggNOG functional profiles for the functional analysis. The gene relative abundance profiles and sequences of integrated gene catalog (IGC) of human gut microbiome [16], and the reference gene catalog of the pig gut metagenome [17], were downloaded and analyzed by the same KEGG and eggNOG functional annotation pipeline in our study.

\section{Microbial composition analysis}

For microbial diversity analysis, Shannon index was used. The overall differences in the bacterial community structures were evaluated by non-metric multidimensional scaling (NMDS) based on Bray-Curtis dissimilarity values and performed with "Phyloseq" package in $\mathrm{R}$.

\section{Co-occurrence network analysis}

We calculated the Spearman's rank correlation coefficient through $\mathrm{R}$ package of "ccrepe" between genera, based on the relative abundance profile of genera. Networks were then constructed by using the method implemented in Cytoscape (v3.6) [82].

\section{Statistical analysis}

The significant functional differences between the chicken, human, and pig gut samples were determined by the Wilcoxon rank-sum test, adjusted by the Storey's methods for multiple tests. Because of the different gene annotation ratios of the chicken, human, and pig gut catalogs, the KEGG functional profiles of the chicken, human, and pig samples were normalized before comparison (genes with no functional annotations were excluded). To avoid the influence of the different intestinal compartments, only when subgroups of the five chicken intestinal compartments all showed the significant difference $(P<0.005)$ compared to the human samples (1267 samples) or pig samples (287 samples), the asterisk was shown in Fig. 1f.

To determine the taxonomic and functional differences between the foregut and hindgut microbial communities, the Wilcoxon rank-sum test (Storey's methods for multiple tests adjustment) was applied. For the comparison of treatment groups, the Kruskal-Wallis test (Storey's methods for multiple tests adjustment) was applied, followed by the post-hoc Wilcoxon rank-sum test.

The data of growth performance and $\mathrm{qPCR}$ were analyzed with one-way ANOVA and Duncan's multiple comparison.

\section{Additional files}

Additional file 1: Table S1. Background information on the chicken samples. (XLSX $34 \mathrm{~kb}$ )

Additional file 2: Figure S1. Rarefaction curves of detected genes from the whole set of 495 samples (Total) and from subgroups of each intestinal compartment (99 samples). D (duodenum), J (jejunum), I (ileum), C (cecum), R (colorectum). The gene number of a specific number of samples was calculated after random samplings repeated 100 times with replacement, and the median was plotted. (PDF $211 \mathrm{~kb}$ )

Additional file 3: Table S2. Comparison of gut microbial gene catalogs of human, pig and chicken. (XLSX 38 kb)

Additional file 4: Table S3. Significant differences between the foregut and hindgut of chickens at the phylum and genus levels. (XLSX $40 \mathrm{~kb}$ )

Additional file 5: Figure S2. Functional annotation of gut microbial genes based on KEGG orthologous groups (KOs) and eggNOG orthologous groups (OGs). (a) Comparison of the total gene numbers and the functionally annotated gene numbers of the chicken, human and pig catalogs. (b) Comparison of the number of KOs and OGs presented in the chicken, human and pig catalogs. (PDF $165 \mathrm{~kb}$ )

Additional file 6: Figure S3. Venn diagram of eggNOG orthologous groups (OGs) presented in and shared by the chicken, human and pig catalogs. (PDF $103 \mathrm{~kb}$ )

Additional file 7: Figure S4. The NMDS plot of the chicken, human and pig gut samples based on Bray-Curtis dissimilarities at $\mathrm{KO}$ level. (PDF 900 kb)

Additional file 8: Figure S5. Microbial community compositions of the duodenum, jejunum, ileum, cecum, and colorectum across 495 chicken gut samples. (a) Phylum-level compositions. (b) Genus-level compositions. "Others" refers to all the other phyla or genera in the samples (unclassified not included). (PDF $429 \mathrm{~kb}$ )

Additional file 9: Figure S6. The NMDS plot of foregut samples in five Distribution groups (DGY, DHC, DHK, DSL, DST) based on Bray-Curtis dissimilarities at the species level. (PDF $887 \mathrm{~kb}$ )

Additional file 10: Figure S7. Microbial diversity (Shannon index) of samples in five Distribution groups (DGY, DHC, DHK, DSL, DST) at gene, genus, $\mathrm{OG}$ and $\mathrm{KO}$ levels. Box plots show median \pm interquartile range $(\mathrm{QQR})$ and $1.5 \mathrm{IQR}$ ranges (whiskers), with outliers denoted by dots. (PDF 866 kb)

Additional file 11: Figure S8. Average relative abundance of phylum Actinobacteria of foregut samples in five Distribution groups (DGY, DHC, DHK, DSL, DST). (PDF 744 kb)

Additional file 12: Table S4. The number of shared genes of samples in each intestinal compartment of chickens. (XLSX $53 \mathrm{~kb}$ )

Additional file 13: Figure S9. Comparison of KEGG functional profiles of five intestinal compartments. The average relative abundance of samples in each KEGG functional category was plotted. (PDF $760 \mathrm{~kb}$ )

Additional file 14: Figure S10. Microbial gene functional differences between foregut and hindgut though eggNOG annotation (Wilcoxon rank-sum test, Storey's methods for multiple tests adjustment). (PDF $812 \mathrm{~kb}$ )

Additional file 15: Figure S11. Comparison of eggNOG functional profiles of five intestinal compartments. The average relative abundance of samples in each eggNOG functional categories was plotted. (PDF 773 kb)

Additional file 16: Figure S12. The NMDS plot of microbial communities in hindgut at different ages. The analysis was based on Bray-Curtis dissimilarities at species level and samples were grouped according to the ages. (PDF $194 \mathrm{~kb}$ )

Additional file 17: Figure S13. Microbial diversity (Shannon index) at gene, genus, OG and $\mathrm{KO}$ levels of hindgut samples at different ages. Box plots show median \pm interquartile range $(\mathrm{IQR})$ and $1.5 \mathrm{IQR}$ ranges (whiskers), with outliers denoted by dots. (PDF $201 \mathrm{~kb}$ ) 
Additional file 18: Table S5. The relative abundances of major phyla at different ages (average relative abundance > 0.01\%). (XLSX $40 \mathrm{~kb}$ )

Additional file 19: Table $S 6$. The relative abundances of major genera at different ages (average relative abundance > 0.01\%). (XLSX 44 kb)

Additional file 20: Figure S14. The relative abundance of major genera in the foregut and hindgut at different ages. The average relative abundance of samples was plotted. (PDF $552 \mathrm{~kb}$ )

Additional file 21: Figure S15. The differences in KEGG functional pathways of the microbiome in the hindgut at different ages. (PDF $172 \mathrm{~kb}$ )

Additional file 22: Figure S16. The NMDS plot of microbial communities in BLANK and MCE groups (left); and in BLANK and CTC (right). The analysis was based on Bray-Curtis dissimilarities at the species level. (PDF 877 kb)

Additional file 23: Figure S17. The influences of CTC and MCE on microbial diversity (Shannon index) at gene, genus, OG and KO levels (a) in the foregut and (b) in the hindgut. Box plots show median \pm interquartile range (IQR) and 1.5 IQR ranges (whiskers), with outliers denoted by dots. Asterisks denote the significant changes (Wilcoxon rank-sum test, $P<0.05$ ) between BLANK and the growth promoter treated group. (PDF $859 \mathrm{~kb}$ )

Additional file 24: Figure S18. (a) The relative abundances of genera increased by MCE or CTC in the foregut. Kitasatospora and Streptomyces were significantly $(P<0.05)$ increased by $C T C$. (b) The relative abundances of antibiotic biosynthesis pathways were significantly $(P<0.05)$ increased by CTC. (c) The relative abundances of antibiotic resistance genes (ARGs). ARGs were increased $(P<0.1)$ by CTC. Box plots show median \pm interquartile range (IQR) and $1.5 \mathrm{IQR}$ ranges (whiskers), outliers denoted by dots. (PDF $957 \mathrm{~kb}$ )

Additional file 25: Figure S19. The relative abundances of four major classes of ARGs changed by MCE or CTC in the foregut. Box plots show median \pm interquartile range (IQR) and $1.5 \mathrm{IQR}$ ranges (whiskers), outliers denoted by dots. (PDF $823 \mathrm{~kb}$ )

Additional file 26: Figure S20. The relative abundances of core genera significantly changed by CTC or MCE in the foregut. Box plots show median \pm interquartile range (IQR) and 1.5 IQR ranges (whiskers), outliers denoted by dots. Asterisks denote the significant changes (Wilcoxon rank-sum test, $P<0.05$ ) between BLANK and the growth promoter treated groups. (PDF $963 \mathrm{~kb}$ )

Additional file 27: Figure S21. The influences of CTC and MCE on the hindgut microbial functions. The heatmap of KEGG metabolic pathways in hindgut (12 samples for each group, including 6 samples from AA chickens and 6 samples from LY chickens). The relative abundance of each pathway was colored according to its row z-score ((value - row mean)/row standard deviation). Black and white rectangles at the right side of the heatmap represent the significant decrease $(P<0.05)$, and no significant change $(P>0.05)$ compared to BLANK, respectively. The Kruskal-Wallis test (Storey's methods for adjustment) was used followed by a post-hoc Wilcoxon rank-sum test. (PDF 256 kb)

Additional file 28: Table S7. KEGG pathways significantly changed in the foregut after CTC and MCE treatment. (XLSX $13 \mathrm{~kb}$ )

Additional file 29: Table S8. The relative mRNA levels of cytokines in the ileum of Arbor Acres broiler chickens on days 21 and 42. (XLSX 11 kb)

Additional file 30: Table S9. Primers used for quantitative real-time PCR. (XLSX 28 kb)

\section{Acknowledgements}

We thank students at the Hunan Agriculture University, especially Mei Chen, Qi Shi, Huancheng Zhang for help with sample collection. Thanks are also due to Shuqu Li and Wanxu Yang for assistance in performing experiments and data analysis, and to Yingying Zhang for assistance with Figs. 1a and 5 editing. We thank William J Lucas, Elaine Lai-Han Leung, and Xing-Xing Fan for assistance with manuscript editing.

\section{Funding}

This project was supported by grants from the National Key R\&D Program of China (2017YFD0501500, 2016YFD0501308 and 2016YFC1200600), the Shenzhen
Science and Technology Program (JCYJ20150630165133395), the Fund of Key Laboratory of Shenzhen (ZDSYS20141118170111640), the Agricultural Science and Technology Innovation Program (ASTIP) of the Chinese Academy of Agricultural Sciences, and the Elite Youth Program of the Chinese Academy of Agricultural Sciences.

\section{Availability of data and materials}

Gut metagenome sequences were deposited in the National Center for Biotechnology Information (NCBI) PRJNA417359. Gene sequences and related relative abundance profiles were uploaded at the AGIS (ftp:// ftp.agis.org.cn/ fanwei/Chicken_gut_metagenome).

\section{Authors' contributions}

$J Z, W F$, and $Y G$ conceived the study and designed the experiments. $P H, Y Z$, $K X, J F, Y L, X H, H L, X L, Z Q, J H, L Y, Q L, C Z, X S, J Y, L L, N H$, and $H C$ collected the samples and performed experiments. PH, YZ, KX, JF, HW, DT, DL, and BL analyzed the data. $C L, Y R$, and $L Y$ provide suggestions and help checking. WF, $\mathrm{PH}, \mathrm{YZ}, \mathrm{KX}, J \mathrm{~F}, \mathrm{HW}, \mathrm{DT}$, and DL wrote the manuscript. JZ, YG, and SH help revise the manuscript. All authors commented on the manuscript. All authors read and approved the final manuscript.

\section{Ethics approval}

This study was approved by the China Agricultural University Animal Care and Use Committee (CAU 20150428-2).

\section{Consent for publication}

Not applicable.

\section{Competing interests}

The authors declare that they have no competing interests.

\section{Publisher's Note}

Springer Nature remains neutral with regard to jurisdictional claims in published maps and institutional affiliations.

\section{Author details}

${ }^{1}$ Hunan Key Laboratory of Traditional Chinese Veterinary Medicine, Hunan Agricultural University, Changsha 410128, Hunan, China. ${ }^{2}$ College of Horticulture and Landscape, Hunan Agricultural University, Changsha 410128, Hunan, China. ${ }^{3}$ Agricultural Genomic Institute, Chinese Academy of Agricultural Sciences, Shenzhen 518120, Guangdong, China. ${ }^{4}$ State Key Laboratory of Animal Nutrition, College of Animal Science and Technology, China Agricultural University, Beijing 100193, China. ${ }^{5}$ College of Veterinary Medicine, Hunan Agricultural University, Changsha 410128, Hunan, China. ${ }^{6}$ College of Animal Science and Technology, Hunan Agricultural University, Changsha 410128, Hunan, China. ${ }^{7}$ National and Local Union Engineering Research Center of Veterinary Herbal Medicine Resource and Initiative, Hunan Agricultural University, Changsha 410128, Hunan, China.

Received: 27 June 2018 Accepted: 2 November 2018 Published online: 27 November 2018

\section{References}

1. World Health Organization. Antimicrobial Resistance: Global Report on Surveillance. Geneva: World Health Organization; 2014.

2. Aarestrup F. Sustainable farming: get pigs off antibiotics. Nature. 2012; 486(7404):465-6.

3. Coates ME, Fuller R, Harrison GF, Lev M, Suffolk SF. A comparison of the growth of chicks in the Gustafsson germ-free apparatus and in a conventional environment, with and without dietary supplements of penicillin. Brit J Nutr. 1963;17:141-50.

4. Cox LM, Yamanishi S, Sohn J, Alekseyenko AV, Leung JM, Cho I, Kim SG, Li $\mathrm{H}$, Gao Z, Mahana D, et al. Altering the intestinal microbiota during a critical developmental window has lasting metabolic consequences. Cell. 2014; 158(4):705-21.

5. Gaskins HR, Collier CT, Anderson DB. Antibiotics as growth promotants: mode of action. Anim Biotechnol. 2002;13(1):29-42.

6. Hill C, Guarner F, Reid G, Gibson GR, Merenstein DJ, Pot B, Morelli L, Canani RB, Flint HJ, Salminen $S$, et al. The international scientific association for probiotics and prebiotics consensus statement on the scope and 
appropriate use of the term probiotic. Nat Rev Gastro Hepat. 2014;11(8): 506-14.

7. Gibson GR, Hutkins R, Sanders ME, Prescott SL, Reimer RA, Salminen SJ, Scott K, Stanton C, Swanson KS, Cani PD, et al. The international scientific association for probiotics and prebiotics (ISAPP) consensus statement on the definition and scope of prebiotics. Nat Rev Gastro Hepat. 2017;14(8):491-502.

8. Gadde U, Kim WH, Oh ST, Lillehoj HS. Alternatives to antibiotics for maximizing growth performance and feed efficiency in poultry: a review. Anim Health Res Rev. 2017;18(1):26-45.

9. Franz C, Bauer R, Carle R, Tedesco D, Tubaro A, Zitterl-Eglseer K. Study on the assessment of plant/herb extracts and their naturally or synthetically produced components as "additives" for use in animal production. EFSA Support Pub. 2007;4(4):297. https://efsa.onlinelibrary.wiley.com/doi/abs/10. 2903/sp.efsa.2007.ZN-001.

10. Kosina P, Walterova D, Ulrichova J, Lichnovsky V, Stiborova M, Rydlova H, Vicar J, Krecman V, Brabec MJ, Simanek V. Sanguinarine and chelerythrine: assessment of safety on pigs in ninety days feeding experiment. Food Chem Toxicol. 2004;42(1):85-91.

11. Lin L, Liu YC, Huang JL, Liu XB, Qing ZX, Zeng JG, Liu ZY. Medicinal plants of the genus Macleaya (Macleaya cordata, Macleaya microcarpa): a review of their phytochemistry, pharmacology, and toxicology. Phytother Res. 2018; 32(1):19-48

12. Kosina P, Gregorova J, Gruz J, Vacek J, Kolar M, Vogel M, Roos W, Naumann K, Simanek V, Ulrichova J. Phytochemical and antimicrobial characterization of Macleaya cordata herb. Fitoterapia. 2010;81(8):1006-12.

13. Zhang X, Zhao YF, Xu J, Xue ZS, Zhang MH, Pang XY, Zhang XJ, Zhao LP. Modulation of gut microbiota by berberine and metformin during the treatment of high-fat diet-induced obesity in rats. Sci Rep. 2015;5:14405.

14. Zhang $X$, Zhao YF, Zhang MH, Pang XY, Xu J, Kang CY, Li M, Zhang CH, Zhang ZG, Zhang YF, et al. Structural changes of gut microbiota during berberine-mediated prevention of obesity and insulin resistance in high-fat diet-fed rats. PLoS One. 2012;7(8):e42529.

15. Qin JJ, Li RQ, Raes J, Arumugam M, Burgdorf KS, Manichanh C, Nielsen T, Pons $N$, Levenez F, Yamada $T$, et al. A human gut microbial gene catalogue established by metagenomic sequencing. Nature. 2010; 464(7285):59-65.

16. Li JH, Jia HJ, Cai XH, Zhong HZ, Feng Q, Sunagawa S, Arumugam M, Kultima $J R$, Prifti E, Nielsen T, et al. An integrated catalog of reference genes in the human gut microbiome. Nat Biotechnol. 2014;32(8):834-41.

17. Xiao L, Estelle J, Kiilerich P, Ramayo-Caldas Y, Xia Z, Feng Q, Liang S, Pedersen $\mathrm{AO}$, Kjeldsen NJ, Liu C, et al. A reference gene catalogue of the pig gut microbiome. Nat Microbiol. 2016;1:16161.

18. Xiao L, Feng Q, Liang SS, Sonne SB, Xia ZK, Qiu XM, Li XP, Long H, Zhang JF, Zhang DY, et al. A catalog of the mouse gut metagenome. Nat Biotechnol. 2015;33(10):1103-8.

19. Ventura M, Canchaya C, Tauch A, Chandra G, Fitzgerald GF, Chater KF, van Sinderen D. Genomics of Actinobacteria: tracing the evolutionary history of an ancient phylura. Microbiol Mol Biol R. 2007;71(3):495-548.

20. Gong JH, Si WD, Forster RJ, Huang RL, Yu H, Yin YL, Yang CB, Han YM. $16 S$ rRNA gene-based analysis of mucosa-associated bacterial community and phylogeny in the chicken gastrointestinal tracts: from crops to ceca. FEMS Microbiol Ecol. 2007;59(1):147-57.

21. Clench MH, Mathias JR. The avian cecum—a review. Wilson Bull. 1995; 107(1):93-121.

22. Gasaway WC, White RG, Dan FH. Digestion of dry matter and absorption of water in the intestine and cecum of rock ptarmigan. Condor. 1976;78(1):77-84

23. Qu A, Brulc JM, Wilson MK, Law BF, Theoret JR, Joens LA, Konkel ME, Angly F, Dinsdale EA, Edwards RA, et al. Comparative metagenomics reveals host specific metavirulomes and horizontal gene transfer elements in the chicken cecum microbiome. PLoS One. 2008;3(8):e2945

24. Oakley BB, Lillehoj HS, Kogut MH, Kim WK, Maurer JJ, Pedroso A, Lee MD, Collett SR, Johnson TJ, Cox NA. The chicken gastrointestinal microbiome. FEMS Microbiol Lett. 2014;360(2):100-12.

25. Hou QC, Kwok LY, Zheng Y, Wang LF, Guo Z, Zhang JC, Huang WQ, Wang $Y X$, Leng $L, L i H$, et al. Differential fecal microbiota are retained in broiler chicken lines divergently selected for fatness traits. Sci Rep. 2016;6:37376

26. Sergeant MJ, Constantinidou C, Cogan TA, Bedford MR, Penn CW, Pallen MJ. Extensive microbial and functional diversity within the chicken cecal microbiome. PLoS One. 2014;9(3):e91941.
27. Walugembe M, Hsieh JC, Koszewski NJ, Lamont SJ, Persia ME, Rothschild MF. Effects of dietary fiber on cecal short-chain fatty acid and cecal microbiota of broiler and laying-hen chicks. Poult Sci. 2015;94(10):2351-9.

28. Xiao Y, Xiang Y, Zhou W, Chen J, Li K, Yang H. Microbial community mapping in intestinal tract of broiler chicken. Poult Sci. 2017;96(5):1387-93.

29. Videnska P, Faldynova M, Juricova H, Babak V, Sisak F, Havlickova H, Rychlik I. Chicken faecal microbiota and disturbances induced by single or repeated therapy with tetracycline and streptomycin. BMC Vet Res. 2013;9:30.

30. van der Wielen PW, Keuzenkamp DA, Lipman LJ, van Knapen F, Biesterveld S. Spatial and temporal variation of the intestinal bacterial community in commercially raised broiler chickens during growth. Microb Ecol. 2002;44(3):286-93.

31. Stanley D, Hughes RJ, Moore RJ. Microbiota of the chicken gastrointestinal tract: influence on health, productivity and disease. Appl Microbiol Biot. 2014:98(10):4301-10.

32. Cross ML. Microbes versus microbes: immune signals generated by probiotic lactobacilli and their role in protection against microbial pathogens. Fems Immunol Med Mic. 2002;34(4):245-53.

33. LeBlanc JG, Milani C, de Giori GS, Sesma F, van Sinderen D, Ventura M. Bacteria as vitamin suppliers to their host: a gut microbiota perspective. Curr Opin Biotech. 2013;24(2):160-8.

34. Louis P, Flint HJ. Diversity, metabolism and microbial ecology of butyrateproducing bacteria from the human large intestine. FEMS Microbiol Lett. 2009;294(1):1-8.

35. Williams BA, Verstegen MW, Tamminga S. Fermentation in the large intestine of single-stomached animals and its relationship to animal health. Nutr Res Rev. 2001;14(2):207-28.

36. Dai ZL, Wu G, Zhu WY. Amino acid metabolism in intestinal bacteria: links between gut ecology and host health. Front Biosci. 2011;16:1768-86.

37. Vital M, Howe AC, Tiedje JM. Revealing the bacterial butyrate synthesis pathways by analyzing (meta) genomic data. MBio. 2014;5(2):e00889-14.

38. Ding J, Dai R, Yang L, He C, Xu K, Liu S, Zhao W, Xiao L, Luo L, Zhang Y, et al. Inheritance and establishment of gut microbiota in chickens. Front Microbiol. 2017:8:1967.

39. Shaufi MAM, Sieo CC, Chong CW, Gan HM, Ho YW. Deciphering chicken gut microbial dynamics based on high-throughput 165 rRNA metagenomics analyses. Gut Pathog. 2015;7:4.

40. Videnska P, Sedlar K, Lukac M, Faldynova M, Gerzova L, Cejkova D, Sisak F, Rychlik I. Succession and replacement of bacterial populations in the caecum of egg laying hens over their whole life. PLoS One. 2014;9(12):e115142

41. Lu JR, Idris U, Harmon B, Hofacre C, Maurer JJ, Lee MD. Diversity and succession of the intestinal bacterial community of the maturing broiler chicken. Appl Environ Microb. 2003;69(11):6816-24.

42. Hu JL, Yu H, Kulkarni RR, Sharif S, Cui SW, Xie MY, Nie SP, Gong J. Modulation of cytokine gene expression by selected Lactobacillus isolates in the ileum, caecal tonsils and spleen of Salmonella-challenged broilers. Avian Pathol. 2015;44(6):463-9.

43. Connerton PL, Richards PJ, Lafontaine GM, O'Kane PM, Ghaffar N, Cummings $\mathrm{NJ}$, Smith DL, Fish NM, Connerton IF. The effect of the timing of exposure to Campylobacter jejuni on the gut microbiome and inflammatory responses of broiler chickens. Microbiome. 2018;6(1):88.

44. Yakhkeshi S, Rahimi S, Naseri KG. The effects of comparison of herbal extracts, antibiotic, probiotic and organic acid on serum lipids, immune response, GIT microbial population, intestinal morphology and performance of broilers. J Med Plant. 2011;10(37):80-95.

45. Karimi M, Foroudi F, Abedini MR. Effect of Sangrovit on performance and morphology of small intestine and immune response of broilers. Biosci Biotechnol Res Asia. 2014;11(2):855-61.

46. Vieira SL, Berres J, Reis RN, Oyarzabal OA, Coneglian JLB, Freitas DM, Pena JEM, Torres CA. Studies with sanguinarine like alkaloids as feed additive in broiler diets. Braz J Poultry Sci. 2008;10(1):67-71.

47. Wang C, Wang MQ, Ye SS, Tao WJ, Du YJ. Effects of copper-loaded chitosan nanoparticles on growth and immunity in broilers. Poultry Sci. 2011;90(10): 2223-8.

48. Choi JH, Lee K, Kim DW, Kil DY, Kim GB, Cha CJ. Influence of dietary avilamycin on ileal and cecal microbiota in broiler chickens. Poultry Sci. 2017;97(3):970-9.

49. Yeoman CJ, Chia N, Jeraldo P, Sipos M, Goldenfeld ND, White BA. The microbiome of the chicken gastrointestinal tract. Anim Health Res Rev. 2012;13(1):89-99. 
50. Neal-McKinney JM, Lu X, Duong T, Larson CL, Call DR, Shah DH, Konkel ME. Production of organic acids by probiotic Lactobacilli can be used to reduce pathogen load in poultry. PLoS One. 2012;7(9):e43928.

51. Martin R, Miquel S, Ulmer J, Kechaou N, Langella P, Bermudez-Humaran LG. Role of commensal and probiotic bacteria in human health: a focus on inflammatory bowel disease. Microb Cell Factories. 2013;12:71.

52. Duncan SH, Louis P, Flint HJ. Lactate-utilizing bacteria, isolated from human feces, that produce butyrate as a major fermentation product. Appl Environ Microb. 2004;70(10):5810-7.

53. Hamer HM, Jonkers D, Venema K, Vanhoutvin S, Troost FJ, Brummer RJ. Review article: the role of butyrate on colonic function. Aliment Pharm Therap. 2008;27(2):104-19.

54. Rettedal E, Vilain S, Lindblom S, Lehnert K, Scofield C, George S, Clay S, Kaushik RS, Rosa AJM, Francis D, et al. Alteration of the ileal microbiota of weanling piglets by the growth-promoting antibiotic chlortetracycline. Appl Environ Microb. 2009;75(17):5489-95.

55. Zhang DY, Ji HF, Liu H, Wang SX, Wang J, Wang YM. Changes in the diversity and composition of gut microbiota of weaned piglets after oral administration of Lactobacillus or an antibiotic. Appl Microbiol Biot. 2016: 100(23):10081-93.

56. Takahashi Y. Genus Kitasatospora, taxonomic features and diversity of secondary metabolites. J Antibiot. 2017;70(5):506-13.

57. Procopio RED, da Silva IR, Martins MK, de Azevedo JL, de Araujo JM. Antibiotics produced by Streptomyces. Braz J Infect Dis. 2012;16(5):466-71.

58. Soares SC, Silva A, Trost E, Blom J, Ramos R, Carneiro A, Ali A, Santos AR, Pinto AC, Diniz C, et al. The pan-genome of the animal pathogen Corynebacterium pseudotuberculosis reveals differences in genome plasticity between the biovar ovis and equi strains. PLoS One. 2013;8(1):e53818.

59. Lavy A, Rusu R, Mates A. Mycobacterium xenopi, a potential human pathogen. Isr J Med Sci. 1992;28(11):772-5.

60. Saint-Cyr MJ, Haddad N, Taminiau B, Poezevara T, Quesne S, Amelot M, Daube G, Chemaly M, Dousset X, Guyard-Nicodeme M. Use of the potential probiotic strain Lactobacillus salivarius SMXD51 to control Campylobacter jejuni in broilers. Int J Food Microbiol. 2017;247:9-17.

61. Torrallardona D, Harris Cl, Fuller MF. Lysine synthesized by the gastrointestinal microflora of pigs is absorbed, mostly in the small intestine. Am J Physiol Endocrinol Metab. 2003;284(6):E1177-80.

62. Konashi S, Takahashi K, Akiba Y. Effects of dietary essential amino acid deficiencies on immunological variables in broiler chickens. Brit J Nutr. 2000; 83(4):449-56.

63. Islam KBMS, Fukiya S, Hagio M, Fujii N, Ishizuka S, Ooka T, Ogura Y, Hayashi T, Yokota A. Bile acid is a host factor that regulates the composition of the cecal microbiota in rats. Gastroenterology. 2011;141(5):1773-81.

64. Jiang CT, Xie C, Li F, Zhang LM, Nichols RG, Krausz KW, Cai JW, Qi YP, Fang ZZ, Takahashi S, et al. Intestinal farnesoid $X$ receptor signaling promotes nonalcoholic fatty liver disease. J Clin Invest. 2015;125(1):386-402.

65. Segain JP, de la Bletiere DR, Bourreille A, Leray V, Gervois N, Rosales C, Ferrier L, Bonnet C, Blottiere HM, Galmiche JP. Butyrate inhibits inflammatory responses through NF kappa B inhibition: implications for Crohn's disease. Gut. 2000;47(3):397-403.

66. Scheppach W. Effects of short chain fatty acids on gut morphology and function. Gut. 1994;35(1 Suppl):S35-8.

67. DeWinne A, Dirinck P. Studies on vitamin E and meat quality .2. Effect of feeding high vitamin E levels on chicken meat quality. J Agr Food Chem. 1996;44(7):1691-6.

68. Boa-Amponsem K, Price SEH, Picard M, Geraert PA, Siegel PB. Vitamin E and immune responses of broiler pureline chickens. Poultry Sci. 2000; 79(4):466-70.

69. Guo ZY, Li JL, Zhang L, Jiang Y, Gao F, Zhou GH. Effects of alpha-lipoic acid supplementation in different stages on growth performance, antioxidant capacity and meat quality in broiler chickens. Brit Poultry Sci. 2014;55(5): 635-43

70. IApajalahti JH, Sarkilahti LK, Maki BR, Heikkinen JP, Nurminen PH, Holben WE. Effective recovery of bacterial DNA and percent-guanine-plus-cytosinebased analysis of community structure in the gastrointestinal tract of broiler chickens. Appl Environ Microbiol. 1998;64(10):4084-8

71. Zoetendal EG, Heilig HG, Klaassens ES, Booijink CC, Kleerebezem M, Smidt H, de Vos WM. Isolation of DNA from bacterial samples of the human gastrointestinal tract. Nat Protoc. 2006;1(2):870-3.

72. Li H, Durbin R. Fast and accurate short read alignment with BurrowsWheeler transform. Bioinformatics. 2009;25(14):1754-60.
73. Li DH, Luo RB, Liu CM, Leung CM, Ting HF, Sadakane K, Yamashita H, Lam TW. MEGAHIT v1.0: a fast and scalable metagenome assembler driven by advanced methodologies and community practices. Methods. 2016:102:3-11.

74. Hyatt D, LoCascio PF, Hauser LJ, Uberbacher EC. Gene and translation initiation site prediction in metagenomic sequences. Bioinformatics. 2012; 28(17):2223-30

75. Li WZ, Godzik A. Cd-hit: a fast program for clustering and comparing large sets of protein or nucleotide sequences. Bioinformatics. 2006;22(13):1658-9.

76. Buchfink B, Xie C, Huson DH. Fast and sensitive protein alignment using DIAMOND. Nat Methods. 2015;12(1):59-60.

77. Gerlach W, Stoye J. Taxonomic classification of metagenomic shotgun sequences with CARMA3. Nucleic Acids Res. 2011;39(14):e91.

78. Kanehisa M, Goto S, Kawashima S, Okuno Y, Hattori M. The KEGG resource for deciphering the genome. Nucleic Acids Res. 2004:32:D277-D80.

79. Huerta-Cepas J, Szklarczyk D, Forslund K, Cook H, Heller D, Walter MC, Rattei T, Mende DR, Sunagawa S, Kuhn M, et al. eggNOG 4.5: a hierarchical orthology framework with improved functional annotations for eukaryotic prokaryotic and viral sequences. Nucleic Acids Res. 2016:44(D1):D286-D93.

80. Jia B, Raphenya AR, Alcock B, Waglechner N, Guo P, Tsang KK, Lago BA, Dave BM, Pereira S, Sharma AN, et al. CARD 2017: expansion and modelcentric curation of the comprehensive antibiotic resistance database. Nucleic Acids Res. 2016;45(D1):D566-D73.

81. Qin JJ, Li YR, Cai ZM, Li SH, Zhu JF, Zhang F, Liang SS, Zhang WW, Guan YL, Shen DQ, et al. A metagenome-wide association study of gut microbiota in type 2 diabetes. Nature. 2012;490(7418):55-60.

82. Shannon P, Markiel A, Ozier O, Baliga NS, Wang JT, Ramage D, Amin N, Schwikowski B, Ideker T. Cytoscape: a software environment for integrated models of biomolecular interaction networks. Genome Res. 2003;13(11):2498-504

\section{Ready to submit your research? Choose BMC and benefit from:}

- fast, convenient online submission

- thorough peer review by experienced researchers in your field

- rapid publication on acceptance

- support for research data, including large and complex data types

- gold Open Access which fosters wider collaboration and increased citations

- maximum visibility for your research: over $100 \mathrm{M}$ website views per year

At $\mathrm{BMC}$, research is always in progress.

Learn more biomedcentral.com/submissions 